\title{
Hiperatividade e déficit de atenção - O tratamento prejudica o crescimento estatural?
}

\author{
Attention deficit disorder and hyperactivity - Does \\ the treatment affect the statural growth?
}

Durval Damiani', Daniel Damiani², Erasmo Casella ${ }^{3}$

1 Unidade de Endocrinologia Pediátrica, Instituto da Criança, Hospital das Clínicas, Faculdade de Medicina, Universidade de São Paulo (ICr-HC-FMUSP), São Paulo, SP, Brasil

${ }^{2}$ Curso de Medicina, Universidade Nove de Julho (Uninove),

São Paulo, SP, Brasil

${ }^{3}$ Unidade de Neurologia Pediátrica, ICr-HC-FMUSP, São Paulo, SP, Brasil

Correspondência para: Durval Damiani Rua Bela Cintra, 2.117, ap. 9 01415-002 - São Paulo, SP, Brasi durvald@iconet.com.br

Recebido em 16/Maio/2009 Aceito em 7/Dez/2009

\section{SUMÁRIO}

O presente estudo avaliou a influência de drogas estimulantes usadas no déficit de atenção e hiperatividade no crescimento estatural. Os autores procederam a uma revisão de literatura coletando artigos publicados sobre déficit de atenção e hiperatividade e sua relação com a baixa estatura. A fonte consultada foi o PubMed e o tópico levantado foi "Crescimento e Metilfenidato"/"Déficit de atenção e hiperatividade versus baixa estatura"/"Metilfenidato e distúrbios de crescimento". Os transtornos de atenção e hiperatividade constituem-se em situações clínicas difíceis, por interferir no bem-estar da criança e no seu relacionamento social, com prejuízos de seu desenvolvimento escolar. Uma vez feito o diagnóstico, as medicações estimulantes como o metilfenidato têm papel primordial no tratamento, mas muito se teme com relação a certos efeitos colaterais, particularmente a perda de peso e a perda estatural. Revisou-se uma série de publicações a respeito e pôde-se verificar que não há consenso sobre tais efeitos colaterais, mas que, mesmo quando ocorrem, não são suficientemente intensos para impedir o tratamento. Um julgamento da relação custo-benefício da medicação é sempre apropriado, mas os benefícios obtidos com a medicação e com a melhora do rendimento escolar e das relações sociais da criança não devem ser esquecidos. Uma cuidadosa monitorização da curva pondoestatural permite que o médico vigie com segurança o tratamento prescrito e possa tomar decisões se julgar que o prejuízo estatural compromete o bem-estar do paciente. Arq Bras Endocrinol Metab. 2010;54(3):262-8

\section{Descritores}

TDAH; metilfenidato; crescimento; baixa estatura; perda de peso

\section{SUMMARY}

The current study evaluated the influence of stimulant drugs used for attention deficit and hyperactivity $(\mathrm{ADH})$ on statural growth. The authors conducted a literature review collecting published articles on attention deficit hyperactivity disorder and its relationship with short stature. The source of information was the PubMed database where the following terms were researched: "Growth and Methylphenidate"/"Attention deficit and hyperactivity versus short stature"/"Methylphenidate and growth disorders". ADH are difficult clinical situations that interfere with the patient's well-being and social and school performance. Once the diagnosis is attained stimulant medications such as methylphenidate have a key role in the treatment but there are concerns regarding their interference in growth and weight gain. We reviewed many publications regarding these side effects and there is no consensus on them; however, even when they happen to occur their intensity is not sufficient to preclude the use of the medication. We have to take into consideration the cost/benefit relationship, remembering that improvement in school and social performance are very welcome to the child and family. Careful monitoring of the growth chart can detect worsening of growth and its intensity will determine if the drug shall or shall not be interrupted. Arq Bras Endocrinol Metab. 2010;54(3):262-8

Keywords

ADDH; methylphenidate; growth; short stature; weight loss 


\section{INTRODUÇÃO}

$\mathrm{A}$ s medicações estimulantes como metilfenidato (MPH), um derivado anfetamínico, constituem-se nos agentes farmacológicos mais utilizados nos transtornos de déficit de atenção e hiperatividade (TDAH) e podem propiciar alívio sintomático importante, com melhora comportamental e melhora de rendimento escolar $(1,2)$.

Como ocorre em todas as situações em que é necessário um tratamento prolongado, o receio do desencadeamento de efeitos colaterais é uma preocupação constante, por parte dos pacientes, familiares e médicos. Por outro lado, o crescimento estatural em nossa sociedade tem assumido uma importância muito grande, havendo certa tendência a se comparar altura a sucesso. A baixa estatura é a causa mais frequente de procura aos endocrinologistas pediátricos. A possibilidade de ocorrer influência sobre o crescimento estatural de crianças com TDAH tratadas tem sido o motivo de maior desconforto em relação à medicação mais utilizada nestes casos, principalmente porque comumente tal terapêutica é introduzida no início da fase escolar e com a expectativa de ser utilizada por longo período de tempo.

Os efeitos do MPH sobre o crescimento foram inicialmente publicados por Safer e cols. $(3,4)$ na década de 1970 e trabalhos posteriores tanto vieram a confirmar alguma interferência no crescimento estatural como outros não observaram diferenças de crescimento em crianças tratadas (5-7).

Objetivou-se, nesta revisão, atualizar os conhecimentos dos efeitos do MPH sobre o crescimento, com o objetivo de permitir ao médico e aos familiares da criança o conhecimento dos vários estudos de caráter científico realizados até o momento. Inicialmente, revisaram-se alguns conceitos do que é o TDAH e de como é efetuado normalmente seu tratamento.

\section{TRANSTORNO DE DÉFICIT DE ATENÇÃO E HIPERATIVIDADE: DIAGNÓSTICO E TERAPÊUTICA}

O TDAH é um distúrbio com bases neurobiológicas $(8,9)$ que se caracteriza pela combinação de déficit de atenção, hiperatividade e impulsividade, sendo o transtorno comportamental mais frequente em crianças. Estima-se que $3 \%$ a $6 \%$ de nossas crianças e adolescentes o apresentem, o que leva a prejuízos no âmbito familiar, escolar e social, bem como na performance acadêmica e no desenvolvimento emocional e afetivo.
O diagnóstico do TDAH é fundamentalmente clínico, baseando-se em critérios operacionais claros e bem definidos, provenientes de sistemas classificatórios como o Diagnostic and Statistical Manual of Mental Health Disorder, Fourth Edition, 2000 (DSM-IV) $(10,11)$ (Tabela 1).

Tabela 1. Critérios Diagnósticos para Transtorno de Déficit de Atenção/ Hiperatividade, de acordo com o DSM-IV

A. Ou (1) ou (2)

(1) seis (ou mais) dos seguintes sintomas de desatenção persistiram por pelo menos 6 meses, em grau mal-adaptativo e inconsistente com o nivel de desenvolvimento:

Desatenção:

(a) frequentemente deixa de prestar atenção a detalhes ou comete erros por descuido em atividades escolares, de trabalho ou outras

(b) com frequência tem dificuldades para manter a atenção em tarefas ou atividades lúdicas

(c) com frequência parece não escutar quando Ihe dirigem a palavra

(d) com frequência não segue instruções e não termina seus deveres escolares, tarefas domésticas ou deveres profissionais (não devido a comportamento de oposição ou incapacidade de compreender instruções)

(e) com frequência tem dificuldade para organizar tarefas e atividades (f) com frequência evita, antipatiza ou reluta a envolver-se em tarefas que exijam esforço mental constante (como tarefas escolares ou deveres de casa)

(g) com frequência perde coisas necessárias para tarefas ou atividades (por exemplo, brinquedos, tarefas escolares, lápis, livros ou outros materiais)

(h) é facilmente distraído por estímulos alheios à tarefa

(i) com frequência apresenta esquecimento em atividades diárias

(2) seis (ou mais) dos seguintes sintomas de hiperatividade persistiram por pelo menos 6 meses, em grau mal-adaptativo e inconsistente com o nível de desenvolvimento:

\section{Hiperatividade:}

(a) frequentemente agita as mãos ou os pés ou se remexe na cadeira

(b) frequentemente abandona sua cadeira em sala de aula ou outras situações nas quais se espera que permaneça sentado

(c) frequentemente corre ou escala em demasia, em situações nas quais isto é inapropriado (em adolescentes e adultos, pode estar limitado a sensações subjetivas de inquietação)

(d) com frequência tem dificuldade para brincar ou se envolver silenciosamente em atividades de lazer

(e) está frequentemente "a mil" ou muitas vezes age como se estivesse "a todo vapor"

(f) frequentemente fala em demasia

\section{Impulsividade:}

(g) frequentemente dá respostas precipitadas antes de as perguntas terem sido completadas

(h) com frequência tem dificuldade para aguardar sua vez

(i) frequentemente interrompe ou se mete em assuntos de outros (por exemplo, intromete-se em conversas ou brincadeiras)

B. Alguns sintomas de hiperatividade-impulsividade ou desatenção que causaram prejuizo estavam presentes antes dos 7 anos de idade

C. Algum prejuízo causado pelos sintomas está presente em dois ou mais contextos (por exemplo, na escola [ou trabalho] e em casa)

D. Deve haver claras evidências de prejuízo clinicamente significativo no funcionamento social, acadêmico ou ocupacional

E. Os sintomas não ocorrem exclusivamente durante o curso de um Transtorno Invasivo do Desenvolvimento, Esquizofrenia ou outro Transtorno Psicótico e não são mais bem explicados por outro transtorno mental (por exemplo, Transtorno do Humor, Transtorno de Ansiedade, Transtorno Dissociativo ou um Transtorno da Personalidade) 
O TDAH pode ser dividido em três tipos: predominantemente hiperativo, predominantemente desatento e combinado. Para o diagnóstico do tipo predominantemente hiperativo, devem estar presentes seis (ou mais) dos nove critérios de hiperatividade do DSM-IV, por um período de pelo menos seis meses, em grau mal-adaptativo e incongruente com o nível de desenvolvimento. $\mathrm{O}$ mesmo deve ocorrer para o diagnóstico do tipo combinado, que deve preencher tanto os critérios de desatenção quanto os de hiperatividade. Os sintomas devem estar presentes em dois ou mais contextos da atividade da vida diária e determinar prejuízos no portador.

De acordo com os conhecimentos adquiridos com base em estudos moleculares, de neuroimagem, neuropsicológicos e farmacológicos, os pacientes com TDAH apresentam alterações reconhecidas em regiões e circuitos cerebrais relacionadas ao controle tanto dos sintomas cardinais (atenção, hiperatividade e impulsividade), como aquelas associadas a um adequado funcionamento executivo, salientando-se estruturas e circuitos associados às regiões pré-frontal dorsolateral, cíngulo cognitivo, loboparietal, gânglios da base e cerebelo (12-14).

Apesar de a neurobiologia do TDAH ainda não ser totalmente compreendida, numerosos estudos apontam para a existência de alterações principalmente nos sistemas noradrenérgicos e dopaminérgicos.

$\mathrm{O}$ tratamento medicamentoso é o de escolha para a maioria dos pacientes com TDAH, determinando acentuada melhora nos sintomas cardinais, que são desatenção, hiperatividade e impulsividade e, consequentemente, melhorando de modo significativo as dificuldades acadêmicas, sociais, familiares e no trabalho dos portadores $(15,16)$. O MPH, que atua inibindo a recaptura da dopamina e da noradrenalina nos neurônios pré-sinápticos, é a primeira opção na terapêutica medicamentosa do TDAH. Isso pode ser claramente demonstrado avaliando-se o tamanho de efeito das diversas possibilidades medicamentosas nessa situação, que é o parâmetro mais adequado para efetuar a comparação entre várias medicações quando não existem estudos suficientemente esclarecedores que avaliem diretamente a eficácia de uma medicação em relação à outra.

Tamanho de efeito de uma medicação refere-se à comparação de sua eficácia em relação ao placebo para o tratamento de determinada doença. Corresponde à diferença entre um grupo de pacientes que recebeu a medicação e outro que recebeu placebo, dividida pelo desvio-padrão combinado desses grupos (17). O tamanho de efeito do metilfenidato variou nos diversos estudos de 0,8 a 1,3 , consideravelmente superior ao de outras medicações disponíveis como a clonidina, a bupropiona e a imipramina que apresentam tamanho de efeito de 0,6 e da atomoxetina de 0,7. O tamanho de efeito igual ou maior que 0,6 é considerado grande, sendo ainda definido como médio quando entre $0,3 \mathrm{e}$ 0,5 e pequeno se menor que 0,3 (17-21).

O MPH de ação imediata, disponível desde 1955, é rapidamente absorvido, apresentando ação terapêutica a partir de 30 minutos, com concentração máxima atingida por volta de 1,5 a 2 horas da administração. $\mathrm{O} \mathrm{MPH}$ é rapidamente metabolizado à sua forma inativa, o ácido ritalínico, o que limita a sua meia-vida a 2 a 3 horas (22-25).

Em decorrência dessas propriedades farmacocinéticas, a ação terapêutica do MPH de ação imediata é de cerca de 4 horas, implicando a necessidade de duas a três administrações ao dia, o que frequentemente prejudica a aderência ao tratamento (23). A dose habitual é $0,3-0,5 \mathrm{mg} / \mathrm{kg} /$ dose, até o limite de $1 \mathrm{mg} / \mathrm{kg} /$ dia, com máximo de $60 \mathrm{mg} /$ dia. Nos Estados Unidos a partir de 2002 e no Brasil desde o ano de 2004, estão disponíveis duas apresentações de longa ação do MPH - MPH-SODAS e o MPH-OROS - com 8 e 12 horas de ação, respectivamente (26-28).

\section{O METILFENIDATO NO CRESCIMENTO - O QUE HÁ NA LITERATURA}

Nas últimas três décadas, repetidamente têm sido levantadas preocupações com relação ao crescimento e ao ganho de peso de crianças e adolescentes que recebem medicações estimulantes. Enquanto os efeitos de tais drogas sobre o peso têm sido mostrados por vários autores, o efeito sobre a altura é menos consistente, havendo estudos mostrando uma desaceleração do crescimento enquanto outros não confirmam tais achados $(5-7,15,29,30)$. Numa revisão de Rapport e Moffitt (31), oito de 11 estudos investigando os efeitos de MPH sobre o peso de crianças em tratamento constataram significantes diferenças nos níveis esperados de ganho de peso, que foi menor no grupo das crianças que utilizaram estimulante em relação ao que receberam placebo ou não foram tratadas. Os demais estudos não documentaram efeito significativo de $\mathrm{MPH}$ no peso, comparado ao basal e aos controles não tratados. Os efeitos sobre a altura foram examinados em 10 estu- 
dos. Quatro mostraram achados significativos, que incluíam redução nos níveis esperados de ganho de altura, que foi menor no grupo dos medicados em relação ao controle. As reduções iniciais no crescimento dos indivíduos tratados com metilfenidato não se mantiveram em dois desses quatro estudos, não se constatando diferenças significativas em relação à altura, nos grupos medicados ou não, no seguimento a longo prazo (31).

No estudo de Charach e cols. (32), os autores avaliam os efeitos sobre o crescimento por um período de um a cinco anos com altas doses de drogas estimulantes. Foram estudadas 89 crianças com TDAH, após um período de 12 meses de tratamento com metilfenidato. As crianças selecionadas tinham de 6 a 12 anos de idade. A dose média de metilfenidato foi de $31,9 \mathrm{mg} /$ dia ou $0,6 \mathrm{mg} / \mathrm{kg} /$ dose, administrada duas vezes ao dia. Ao final de 12 meses, 58 crianças estavam utilizando medicação estimulante e 31 não estavam. Aos cinco anos, 68 crianças permaneciam em seguimento. O estudo demonstrou risco leve, mas existente, de diminuição da taxa de crescimento em pacientes em uso de medicamentos estimulantes, em período prolongado. O risco de redução da taxa de crescimento não se torna estatisticamente significante até a dose de metilfenidato $\geq 2,5 \mathrm{mg} / \mathrm{kg} /$ dia, por mais de quatro anos. As estimativas para alteração de crescimento após uso prolongado revelam um padrão dose-dependente, com os efeitos iniciais, em menores doses, na taxa de ganho de peso, mais do que no ganho estatural. Talvez esse efeito de dose possa explicar alguns resultados controversos com o uso de metilfenidato: os que usam doses maiores mostram alguma interferência no crescimento, enquanto outros, que usam doses menores, não mostram. Estimativas teóricas a partir desse trabalho mostram que doses de $\mathrm{MPH}$ equivalentes a $1,5 \mathrm{mg} / \mathrm{kg} /$ dia poderiam reduzir o ganho de peso em aproximadamente 1,4 kg para um menino de 9 anos, durante um ano de tratamento. Uma dose de $2,5 \mathrm{mg} / \mathrm{kg} /$ dia poderia reduzir o ganho de peso em $2,9 \mathrm{~kg}$. Num período acima de quatro anos de uso, $2,5 \mathrm{mg} / \mathrm{kg} /$ dia poderia reduzir o ganho em altura em $1,9 \mathrm{~cm}$ para um menino de 13 anos de idade. Por outro lado, no trabalho de Zachor e cols. (33), com 89 crianças com TDAH tratadas com medicações psicoestimulantes, o seguimento foi por três anos. Embora o z score de peso tenha mostrado alterações significativas após dois anos de tratamento, as análises posteriores não atingiram significado estatístico. $\mathrm{O}$ índice de massa corpórea esteve dentro dos limites normais durante toda a duração do tratamento.
As crianças pré-púberes eram mais sujeitas à perda de peso do que as crianças em puberdade. Nenhum impacto a longo prazo sobre a estatura foi notado e o uso de diferentes medicações psicoestimulantes não mostrou efeitos diferentes sobre a altura.

Estudos transversais mostram que a estatura de adultos tratados com estimulantes na infância não era significantemente diferente dos controles. Portanto, em algum momento, poderia ter ocorrido o "catch $u p$ " (6), estando de acordo com a possibilidade de que, se ocorre algum efeito no crescimento dos pacientes medicados, ele é limitado aos primeiros anos da terapêutica e a "deficiência estatural" seria espontaneamente recuperada ao final da puberdade.

Spencer e cols. (34), avaliando 178 crianças em tratamento para TDAH, observaram um crescimento a menos de $0,23 \mathrm{~cm}$ no grupo de pacientes medicados, ao final de pelo menos 21 meses de seguimento. Swanson e cols. (35), ao avaliar pré-escolares medicados com estimulante por 12 meses, observaram um menor crescimento em relação ao grupo não medicado de $1,38 \mathrm{~cm}$.

$\mathrm{O}$ estudo mais importante a respeito da terapêutica do TDAH foi o Multimodal Treatment Study (MTA Study) (36), que analisou, por 14 meses de modo randomizado e cego, 579 pacientes com TDAH, de 7 a 9 anos. No final desse período, foi constatado menor crescimento no grupo dos pacientes tratados com medicação de $2,3 \mathrm{~cm}$ em relação aos pacientes tratados sem utilização de medicação. Essas crianças permaneceram em seguimento desde então, tendo já sido efetuadas mais duas publicações a respeito do crescimento estatural, com 24 e 36 meses de seguimento $(37,38)$; observou-se, ao final desses períodos, um menor crescimento nos pacientes medicados com estimulante de $0,67 \mathrm{~cm}$ no intervalo entre 14 e 24 meses de seguimento e, ao final de 36 meses, um crescimento a menos de $2 \mathrm{~cm}$ nos pacientes medicados $(\mathrm{n}=70)$ em relação aos não medicados $(\mathrm{n}=65)$.

Swanson (39) apresentou, no $161^{\circ}$ Meeting da Academia Americana de Psiquiatria em maio de 2008, os resultados de 10 anos de seguimento desses pacientes $\mathrm{e}$, ao comparar os pacientes que receberam medicação por todo o período em relação aos não medicados todo o tempo, constatou-se que o déficit de crescimento que muitos autores relataram deixar de existir com a persistência da terapêutica não tinha ocorrido.

Salienta-se que os resultados obtidos pelo MTA Study - apesar de ter sido em sua primeira publicação o estudo mais importante em relação à terapêutica do 
TDAH, quando ainda era um estudo cego e randomizado - perdeu muito de sua validade, com a possibilidade de migração dos pacientes para os vários grupos analisados, após o período inicial de 14 meses, de acordo com os resultados ou preferência das famílias. Certamente, criou-se um viés que deve ser muito considerado sempre que analisarmos as publicações subsequentes, já que provavelmente foi criado um grupo de pacientes mais graves tratado com medicação todo o tempo e um grupo de pacientes de menor acometimento que permaneceu sem medicação desde o início do estudo.

Findling (40), também durante o 161ํㅡeeting da Academia Americana de Psiquiatria, apresentou os resultados de crianças de 6 a 12 meses tratadas com metilfenidato de liberação lenta ( $p a t c h)$, não observando diferenças no crescimento entre os grupos tratados ou não.

Inferências a respeito dos possíveis mecanismos associados à interferência (pelo menos nos primeiros anos de uso) podem ser efetuadas, como a diminuição do apetite e/ou sono que a medicação pode acarretar. Outra possibilidade a ser discutida seria ainda um próprio crescimento menor dos pacientes com TDAH que poderia ocorrer, de modo análogo ao menor tamanho de áreas do parênquima encefálico nos portadores desse distúrbio (13).

Bossé e cols. (41) especulam a respeito de que o aumento da dopamina nas sinapses determinada pelo metilfenidato, que ocorre principalmente no striatum, por meio do bloqueio da DAT, também poderia determinar aumento de dopamina em outras regiões, como a região hipotalâmica, e que tal fato seria o responsável pelo menor crescimento desses pacientes.

A possibilidade de o aumento da atividade dopaminérgica pelo $\mathrm{MPH}$, utilizado cronicamente, determinar diminuição da secreção de $\mathrm{GH}$ foi demonstrada em dois estudos $(42,43)$, porém outros oitos estudos não evidenciaram qualquer correlação (44-51). Talvez essa interferência na secreção de GH devesse ser considerada quando se analisam casos de baixa estatura idiopática ou outras indicações de uso de $\mathrm{GH}$ em que a causa não fica bem esclarecida (52).

Um estudo realizado em ratos e mais dois outros em humanos sugerem que as alterações hormonais são transitórias e que ocorre normalização do GH e da somatomedina $\mathrm{C}$ durante a manutenção por tempo mais prolongado do tratamento medicamentoso com $\mathrm{MPH}$ $(49,53,54)$.

De acordo com esses dados, neste momento não se pode ainda ter conclusões totalmente definitivas a respeito do efeito do MPH sobre o crescimento dos pacientes com TDAH e que os médicos devem discutir com os familiares a possibilidade de que o metilfenidato possa eventualmente determinar algum tipo de interferência com o crescimento e ponderar em cada caso os riscos da ausência do tratamento medicamentoso em relação a uma eventual menor estatura final da criança.

\section{CONCLUSÃO}

Foram revistos os estudos mais importantes publicados ou divulgados até o momento e a ideia é que se está ainda diante de um fato não resolvido, que é a questão de haver ou não um prejuízo no crescimento dos pacientes medicados quanto ao déficit estatural. Os autores do presente estudo acreditam que, se isso ocorre, não parece ser tão significativo e que a opção da medicação para os pacientes acometidos não deveria, de modo geral, deixar de ocorrer por esse motivo, já que se está perante um distúrbio que, quando não adequadamente abordado, implica prejuízos imediatos e tardios aos portadores. O ideal é que os pacientes sejam monitorizados no tocante ao crescimento e que aqueles que eventualmente possam estar tendo alguma repercussão mais importante sejam abordados por um endocrinologista pediátrico, a fim de que se decida qual a melhor solução para não haver prejuízo estatural.

Declaração: os autores declaram não haver conflitos de interesse científico neste estudo.

\section{REFERÊNCIAS}

1. Swanson JM, Lerner MA, Gupta S, Shoulson I, Wigal S. Development of a new once-a-day formulation of methylphenidate for the treatment of ADHD. Arch Gen Psychiatry. 2003;60:204-11.

2. SpencerTJ, Faranoe SV, Biederman J, Lerner M, Cooper KM, Zimmerman B. Does prolonged therapy with a long-acting stimulant suppress growth in children sith ADHD? J Am Acad Child Adolesc Phychiatry. 2006;45:527-37.

3. Safer DJ, Allen RP. Factors influencing the suppressant effects of two stimulant drugs on the growth of hyperactive children. Pediatrics. 1973;51:660-7.

4. Safer DJ, Allen RP, Barr E. Growth revound after termination of stimulant drugs. J Pediatr. 1975;86:113-6.

5. Gittelman R, Mannuzza S. Hyperactive boys almost grown up III: methylphenidate effects on ultimate height. Arch Gen Psychiatry. 1988;45:1131-4.

6. Kramer JR, Loney J, Ponto LB, Roberts MA, Grossman S. Predictors of adult height and weight in boys treated with methylphenidate for childhood behavior problems. J Am Acad Child Adolesc Psychiatry. 2000;39:517-24.

7. Pliszka SR, Matthews TL, Braslow KJ, Watson MA. Comparative effects of methylphenidate and mixed salts amphetamine on 
height and weight in children with attention-deficit/hyperactivity disorder. J Am Acad Child Adolesc Psychiatry. 2006;45(5):520-6.

8. Shaw P, Eckstrand K, SharpW, Blumenthal J, Lerch JP, Greenstein D, et al. Attention-deficit/hyperactivity disorder is characterized by a delay in cortical maturation. PNAS. 2007;104:19649-654.

9. Barkley RA. Transtorno de déficit de atenção/hiperatividade. Manual para diagnóstico e tratamento associados. 3.ed. Porto Alegre: Artmed; 2008. p. 134-95.

10. America Psychiatric Association Diagnostic and statistical manual of mental disorders 4th ed Washington, DC: American Psychiatric Association; 1994. p 78-85.

11. American Academy of Pediatrics. Clinical practice guideline: diagnosis and evaluation of the child with attention-deficit/hyperactivity disorder. Pediatrics. 2000;105:1158-70.

12. Shaw $P$, Eckstrand $K$, SharpW, Blumenthal J, Lerch JP, Greenstein $D$, et al. Attention-deficit/hyperactivity disorder is characterized by a delay in cortical maturation. PNAS. 2007;104:19649-654.

13. Valera EM, Faraone SV, Murray KE, Seidman LJ. Meta-analysis of structural imaging findings in attention-deficit/hyperactivity disorder. Biol Psychiatry. 2007;61(12):1361-9.

14. Makris N, Buka SL, Biederman J, Papadimitriou GM, Hodge SM, Valera EM, et al. Attention and executive systems abnormalities in adults with childhood ADHD: a DT-MRI study of connections. Cereb Cortex. 2008;18(5):1210-20.

15. Faraone SV, Spencer T, Aleardi M, Pagano C, Biederman J. Meta-analysis of the efficacy of methylphenidate for treating adult attention-deficit/hyperactivity disorder. J Clin Psychopharmacol. 2004;24(1):24-9.

16. Spencer T, Biederman J, Wilens T. Pharmacotherapy of attention deficit hyperactivity disorder. Child Adolesc Psychiatr Clin North Am. 2000;9:77-97.

17. Rosenthal R, Rubin DB. Meta-analytic procedures for combining studies with multiple effect sizes. Psychol Bull. 1986;99:400-6.

18. Connor DF, Fletcher KE, Swanson JM. A meta-analysis of clonidine for symptoms of attention-deficit hyperactivity disorder. J Am Acad Child Adolesc Psychiatry. 1999;38(12):1551-9.

19. Wilens TE, Haight BR, Horrigan JP, Hudziak JJ, Rosenthal NE, Connor DF, et al. Bupropion $\mathrm{XL}$ in adults with attention-deficit/ hyperactivity disorder: a randomized, placebo-controlled study. Biol Psychiatry. 2005;57(7):793-801.

20. Mészáros A, Czobor P, Bálint S, Simon V, Bitter I. [Pharmacotherapy of adult Attention Deficit/Hyperactivity Disorder (ADHD): a systematic review]. Psychiatr Hung. 2007;22(4):259-70.

21. Van der Oord S, Prins PJ, Oosterlaan J, Emmelkamp PM. Efficacy of methylphenidate, psychosocial treatments and their combination in school-aged children with ADHD: a meta-analysis. Clin Psychol Rev. 2008;28(5):783-800.

22. Kimko HC, Cross JT, Abernethy DR. Pharmacokinetics and clinical effectiveness of methylphenidate. Clin Pharmacokinet. 1999;14:457-70.

23. Pelham WE, Gnagy EM, Burrows-Maclean L, Williams A, Fabiano GA, Morrisey SM, et al. Once-a-day Concerta methylphenidate versus three-times-daily methylphenidate in laboratory and natural settings. Pediatrics. 2001;107(6):E105.

24. Swanson JM, Lerner M, WigalT, Steinhoff K, Greenhill L, Posner K, et al. The use of a laboratory school protocol to evaluate concepts about efficacy and side effects of new formulations of stimulant medications. J Atten Disord. 2002;6(suppl 1):S73-88.

25. Markowitz JS, Straughn, AB, Patrick KS. Advances in the pharmacotherapy of ADHD: focus on methylphenidate formulations. Pharmacotherapy. 2003;23;10:1281-99.

26. Gonzalez MA, Pentikis HS, Andrl N, Benedict MF, DeCory HH, Hirshey-Dirksen SJ, et al. Methylphenidate bioavailability from two extended-release formulations. Int $\mathrm{J}$ Clin Pharmacol Ther. 2002;40(4):175-84.
27. Greenhill LL, Findling RL, Swanson JM; the MPH MR ADHD Study Group. A double-blind, placebo-controlled study of modified-release methylphenidate in children with Attention-Deficit/Hyperactivity Disorder. Pediatrics. 2002;109(3):E39.

28. Banaschewski T, Coghill D, Santosh $P$, et al. Long-acting medications for the hyperkinetic disorders. A systematic review and European treatment guideline. Eur Child Adolesc Pscyhiatry. 2006;15:476-95.

29. Remschmidt $H$, Hoare $P$, Ettrich $C$, Tothenberger $A$, Santosh $P$, Schmidt $M$, et al. Symptom control in children and adolescents with ADHD on switching from immediate-release MPH to OROS. Results of a 3-week open label study. Eur Child Adolesc Psychiatry. 2005;14:297-301.

30. Vincent J, Varley C, Leger P. Effects of methylphenidate on early adolescent growth. Am J Psychol. 1990;147:501-2.

31. Rapport MD, Moffitt C. Attention deficit/hyperactivity disorder and methylphenidate. A review of height/weight, cardiovascular, and somatic complaint side effects. Clin Psychol Rev. 2002;22:1107-31.

32. Charach A, Figueroa M, Chen S, Ickowicz A, Schachar R. Stimulant treatment over 5 years: effects on growth. J Am Acad Child Adolesc Psychiatry. 2006;45(4):415-21.

33. Zachor DA, Roberts AW, Hodgens JB, Isaacs JS, Merrick J. Effects of long-term psychostimulant medication on growth of children with ADHD. Res Dev Disabil. 2006;27:162-74.

34. SpencerT, Biederman J, Ciccone PE, Madras BK, Dougherty DD, Bonab AA, et al. PET study examining pharmacokinetics, detection and likeability, and dopamine transporter receptor occupancy of short- and long-acting oral methylphenidate. Am J Psychiatry. 2006:163:387-95.

35. Swanson J, Greenhill L, Wigal T, Kollins S, Stehli A, Davies M, et al. Stimulant-related reductions of growth rates in the PATS. J Am Acad Child Adolesc Psychiatry. 2006;45(11):1304-13.

36. The MTA Cooperative Group. A 14-month randomized clinical trial of treatment strategies for attention-deficit/hyperactivity disorder. MultimodalTreatment Study of Children with ADHD. Arch Gen Psychiatry. 1999;56(12):1073-86.

37. MTA Cooperative Group. National Institute of Mental Health Multimodal Treatment Study of ADHD follow-up: changes in effectiveness and growth after the end of treatment. Pediatrics. 2004:113:762-9

38. Jensen PS, Arnold LE, Swanson JM, Vitiello B, Abikoff HB, Greenhill LL, et al. 3-year follow-up of the NIMH MTA study. J Am Acad Child Adolesc Psychiatry. 2007;46(8):989-1002.

39. Swanson JM. 161st Annual Meeting of the American Psychiatric Association (APA). Conference: Long-Term effects of stimulants in children with ADHD.

40. Findling R. Effects of methylphenidate transdermal system (MTS) on growth in children with ADHD. 161st Annual Meeting of the American Psychiatric Associations (APA) (poster session) (Abstract NR6-027)

41. Bossé R, Fumagalli F, Jaber M, Giros B, Gainetdinov RR, Wetsel $\mathrm{WC}$, et al. Anterior pituitary hypoplasia and dwarfism in mice lacking the dopamine transporter. Neuron. 1997;19(1):127-38.

42. Aarskog D, Fevang F, Klove H, Stoa F, Thorsen T. The effect of the stimulant drugs, dextroamphetamine and methylphenidate, on secretion of growth hormone in hyperactive children. Pediatrics. 1977; $90: 136-9$

43. Hunt RD, Cohen DJ, Anderson G, Clark L. Possible change in noradrenergic receptor sensitivity following methylphenidate treatment: growth hormone and MHPG response to clonidine challenge in children with attention deficit disorder and hyperactivity. Life Sci. 1984;35:885-97.

44. Greenhill LL, Puig-Antich J, Chambers W, Rubinstein B, Halpern F, Sachar EJ. Growth hormone, prolactin, and growth responses 
in hyperkinetic males treated with D-amphetamine. J Am Acad Child Psychiatry. 1981;20:84-103.

45. Schultz F, Hayford J, Wolraich M, Hintz R, Thompson R. Methylphenidate treatment of hyperactive children: effects on the hypothalamic-pituitary-somatomedin axis. J Pediatr. 1982;70:987-92.

46. Shaywitz SE, Hunt RD, Jatlow $P$, et al. Psychopharmacology of attention deficit disorder: pharmacokinetic, neuroendocrine, and behavioral measures following acute and chronic treatment with methylphenidate. Pediatrics. 1982;69:688-94.

47. Garfinkel B, Brown W, Klee S, Braden W, Beauchesne R, Shapiro S. Neuroendocrine and cognitive responses to amphetamine in adolescents with a history of attention deficit disorder. J Am Acad Child Adolesc Psychiatry. 1986;25:503-8.

48. Weizman R, Dick J, Gil Al, Weitz R, Tyano S, Laron Z. Effects of acute and chronic methylphenidate administration on beta-endorphin, growth hormone, prolactin and cortisol in children with attention deficit disorder and hyperactivity. Life Sci. 1987;40:2247-52.

49. Bereket A, Turan S, Karaman MG, Haklar G, Ozbay F, Yazgan MY. Height, weight, IFG-I, IGFBP-3 and thyroid functions in prepuber- tal children with attention deficit hyperactivity disorder: effect of methylphenidate treatment. Horm Res. 2005;63:159-64.

50. Greenhill LL, Puig-Antich J, Novacenko MA, Solomon M. Prolactin, growth hormone and growth responses in boys with attention deficit disorder and hyperactivity treated with methylphenidate. J Am Acad Child Psychiatry. 1984;23:58-67.

51. Toren $P$, Silbergeld A, Eldar $S$, et al. Lack of effect of methylphenidate on serum growth hormone (GH), GH-binding protein, and insulin-like growth factor 1. Clin Neuropharmacol. 1997;20:264-9.

52. Longui $\mathrm{CA}$. Uso de $\mathrm{GH}$ em pacientes com baixa estatura idiopática. Arq Bras Endocrinol Metabol. 2008;52:750-6.

53. Thanos PK, Michaelides M, Benveniste H, Wang GJ, Volkow ND. Effects of chronic oral methylphenidate on cocaine self-administration and striatal dopamine D2 receptors in rodents. Pharmacol Biochem Behav. 2007;87:426-33.

54. Adriani W, Leo D, Guarino M, et al. Short-term effects of adolescent methylphenidate exposure on brain striatal gene expression and sexual/endocrine parameters in male rats. Ann NY Acad Sci. 2006;1074:52-73. 